\title{
SOME REMARKS ON INFINITESIMAL GENERATORS OF ANALYTIC SEMIGROUPS
}

\author{
JEROME A. GOLDSTEIN ${ }^{1}$
}

It is well known that many infinitesimal generators of strongly continuous one parameter semigroups of bounded linear operators on a Banach space actually generate semigroups which are analytic in a sector $\Sigma(\theta)=\{z:|\arg z|<\theta\}$ where $0<\theta \leqq \pi / 2$ (see [4, p. 260]). However, the infinitesimal generator of an analytic group is necessarily a bounded operator. In fact, the following stronger result is valid.

Proposition 1. Let $A$ be the infinitesimal generator of a semigroup which is analytic in $\Sigma(\theta)$ where $0<\theta \leqq \pi / 2$. If, in addition, $-A$ is the infinitesimal generator of a strongly continuous semigroup, then $A$ is a bounded operator.

Proof. If $A$ generates a semigroup analytic in $\Sigma(\theta)$, then $A e^{t A}$ is a bounded operator for each $t>0$ by $\left[4\right.$, pp. 254-255], where $\left\{e^{t A}\right\}$ denotes the semigroup generated by $A$. If also $-A$ generates a semigroup, then $A=\left(A e^{t A}\right)\left(e^{-t A}\right)$ is bounded, being a composition of bounded operators. We note that by $[4, \mathrm{pp} .254-255]$, the above proposition is true if the Banach space is replaced by a sequentially complete locally convex topological vector space and the term "bounded operator" is replaced by "continuous operator."

An alternate proof can be obtained with the aid of standard results on the generation of semigroups (namely, Proposition 3 below and the Hille-Yosida Theorem) by noting that the hypotheses imply that the spectrum of $A$ is a bounded subset of the complex plane and

$$
\left\|(\lambda-A)^{-1}\right\|=O(1 /|\lambda|)
$$

as $|\lambda| \rightarrow \infty$. It then follows by [1, Lemma 5.2] that $A$ is a bounded operator.

Corollary 1. Let $A$ generate a semigroup analytic in $\Sigma(\theta)$ for some $\theta>0$. Then the backward Cauchy problem

$$
u^{\prime}(t)=A u(t) \quad(-\infty<t \leqq 0), \quad u(0)=f
$$

(where' $=d / d t)$ is well posed if and only if $A$ is bounded.

This is an immediate consequence of the proposition.

\footnotetext{
Received by the editors September 30, 1968.

${ }^{1}$ Partially supported by Air Force Office of Scientific Research Grant AF-AFOSR-
} $359-66$. 
In [2] it was proved that if the Cauchy problem

$$
u^{\prime \prime}(t)=A u(t) \quad(-\infty<t<\infty), \quad u(0)=f, \quad u^{\prime}(0)=g
$$

is well posed, then $A$ is the infinitesimal generator of a semigroup analytic in $\Sigma(\pi / 2)$.

An immediate consequence of this fact and the above corollary is the following result:

CoROllaRy 2. If (2) is well posed, then the backward problem (1) is well posed if and only if $A$ is bounded.

The next result shows that if $A$ is the infinitesimal generator of a semigroup analytic in the right half-plane, then so is $-A^{2}$. It follows from this and the result of [2] mentioned above that $(-1)^{n} A^{2 n}$ is the infinitesimal generator of a semigroup analytic in the right half-plane if (2) is well posed.

Proposition 2. Let $A$ be the infinitesimal generator of a semigroup analytic in $\Sigma(\theta)$ where $\theta>\pi / 4$. Then $-A^{2}$ is the infinitesimal generator of a semigroup analytic in $\Sigma(2 \theta-\pi / 2)$.

The proof is based on the following result:

Proposition 3. A necessary and sufficient condition that a linear operator $A$ be the infinitesimal generator of a semigroup $\{T(t), t \in \Sigma(\theta)\}$ $(0<\theta \leqq \pi / 2)$ such that

(i) $T(t) T(s)=T(t+s)$ for all $t, s \in \Sigma(\theta)$,

(ii) $t \rightarrow T(t)$ is analytic in $\Sigma(\theta)$,

(iii) $\sup \left\{\left\|T\left(r e^{i \alpha}\right)\right\|: 0<r \leqq 1\right\}<\infty$ for $|\alpha|<\theta$,

(iv) $T(t) \rightarrow I$ in the strong operator topology as $|t| \rightarrow 0$ in $\Sigma(\theta-\epsilon)$ for each $\epsilon>0$

is that

(v) $A$ is closed and densely defined,

(vi) for each $\epsilon>0$ there are constants $M_{\epsilon}, C_{\epsilon}$ such that $\lambda$ is in the resolvent set of $A$ and $\left\|(\lambda-A)^{-1}\right\| \leqq M_{\epsilon} /|\lambda|$ whenever $|\lambda| \geqq C_{\epsilon}$ and $\lambda \in \Sigma(\theta-\epsilon+\pi / 2)$.

This is essentially Hille's well-known theorem on the generation of analytic semigroups and can be proved by modifying Hille's proof [3, pp. 384-386].

Proof of Proposition 2. Let $\epsilon>0$ be fixed but arbitrary. Then by Proposition 3, there are constants $C_{\epsilon}, M_{\epsilon}$ such that

$$
\left\|(\lambda-A)^{-1}\right\| \leqq M_{\epsilon} /|\lambda|
$$

whenever $|\lambda| \geqq C_{\mathrm{e}}$ and $\lambda \in \Sigma(\theta-\epsilon+\pi / 2)$. For $\lambda \notin(-\infty, 0]$ let $\mu$ be 
the square root of $\lambda$ with positive real part. Then

$$
\left\|\left(\lambda+A^{2}\right)^{-1}\right\|=\left\|(i \mu-A)^{-1}(-i \mu-A)^{-1}\right\| \leqq M_{\epsilon}^{2} /|\lambda|
$$

whenever $\pm i \mu \in \Sigma(\theta-\epsilon+\pi / 2)$ and $|\mu| \geqq C_{\epsilon}$, that is, whenever $\lambda \in \Sigma(2 \theta-2 \epsilon)$ and $|\lambda| \geqq C_{\epsilon}^{2}$. Hence by Proposition $3,-A^{2}$ is the infinitesimal generator of a semigroup analytic in $\Sigma(2 \theta-\pi / 2)$.

\section{REFERENCES}

1. J. A. Goldstein, Semigroups and hyperbolic equations, J. Functional Analysis 3 (1969).

2. - On a connection between first and second order equations in Banach spaces, (to appear).

3. E. Hille and R. S. Phillips, Functional analysis and semigroups, Amer. Math. Soc. Colloq. Publ., vol. 31, Amer. Math. Soc., Providence, R. I., 1957.

4. K. Yosida, Functional analysis, Springer, Berlin, 1965.

THE INSTITUTE For Advanced StUdy AND

TUlane University 